\section{Tracking Trains via Radio Frequency Systems}

António José Duarte Santos, Américo Rodrigues Soares, Fernando Manuel de Almeida Redondo, and Nuno Borges Carvalho

\begin{abstract}
The technological evolution which occurred in digital systems favored the appearance of new services to be applied in railways. Radio communications played an important role in the management, exploration, and maintenance of railway transports. Due to the vital importance of security, the track-to-train communications system is studied in depth, with significant changes through the integration in its operation of the global system for mobile communication and global positioning systems. This paper presents an integrated system that simultaneously includes a radio communication system and a location solution. The proposed network will work as a redundant and also as a unique system for secondary lines where no other system is available. Moreover, the paper also presents a low-budget system to track the trains inside tunnels.
\end{abstract}

Index Terms-Global positioning system, road vehicle location monitoring.

\section{INTRODUCTION}

A large part of the public transport management budget is spent in safety. In railway companies, this fact becomes especially relevant, meaning that the correct train management and location is fundamental. Several tracking systems have been used, although with high costs associated, since these usually imply physical changes in the full network. With the advent of telecommunications, especially with radio communications, new systems surfaced, allowing the implementation of train tracking and thus increasing safety, especially due to the possibility of data transmission between the driver and the regulator. Recently, there has been a large proliferation of radio systems for public use, and its capacities and range also expanded. An example is the global system for mobile communication (GSM) network. Therefore, the next logical step to increase the number of services is to use a GSM equivalent system.

Two main points are of specific concern: the transition in real systems such as these cannot be done abruptly, and also due to the public nature of the actual GSM network, meaning that the responsibility for train safety cannot be completely passed on to external entities.

Currently, the communication systems are mainly proprietary, as for example the current communication system in Portuguese railways which is a private cellular system, known as track-to-train communications [1] that allows data and voice communications. Track-to-train [Rádio Solo Comboio (RSC)] is a transmission system used in the communication between the traffic regulator agent and the drivers aiming to send auxiliary operation information. For that purpose, the railway line is divided in sections, which are known as regulation sectors. An independent track-to-train system operates in each regulation sector.

Due to the system's concept, it is only possible to establish only one telephony communication at a time. The regulator agent ensures the

Manuscript received February 18, 2004; revised November 30, 2004. The Associate Editor for this paper was F.-Y. Wang.

A. J. D. Santos and N. B. Carvalho are with the Instituto de Telecomunicacoes, University of Aveiro, Aveiro, Portugal (e-mail: nborges@ieee.org).

A. R. Soares is with NEC Portugal, Monte de Caparica, Portugal.

F. M. de Almeida Redondo is with CP-Portuguese Railways, Lisbon, Portugal.

Digital Object Identifier 10.1109/TITS.2005.848369 management of the communications priority. It is also possible to establish communications via a telephone network to the inside of the train, and vice versa, using a voice channel. This type of communication always goes through the regulator agent who authorizes it, who grants it priority order, and who also carries out the switch necessary for the connection between both lines. This feature is currently used only for communication between the traction permanent and the drivers. The voice channel may send an alarm message started by any agent (regulator, driver, or station chief). That message overlaps the other communications and is received by all stations. The information debit of track-to-train communications allows data transmission, but with a very limited capacity. Presently, the track-to-train allows voice communications and also sends numerical information data regarding train speed, state of the automatic train control (ATC), base station, etc. This data will be received in a central computer used for the purpose and shared through the internal computer network by the interested parties.

The simple signaling systems using the communication between the control station and the driver, based on the European train control system (ETCS), which should be applied in certain Portuguese railways lines, require as support a transmission system with a debit higher than the track-to-train one. Besides a voice channel, multiple transmissions of data from the control station to the driver's cabin should also be considered when these are running and vice versa.

This scenario leads to a simultaneous message sending through different channels, and to a high amount of message sending per each channel. The communication system to be used should allow for safe data transmission, not cause delays, and also guarantee a noncongested sending of all necessary messages. These prerogatives are fulfilled through the use of a GSM public network, in the short message service (SMS) modality evolving to the use of a dedicated GSM network called GSM for railway applications (GSM-R) [2].

In this paper, the integration of the track-to-train communications with the public GSM network will be presented. This will allow not only to have a redundant system in lines where we already have the track-to-train infrastructure but also in secondary lines where no radio communication system exists.

In the case that the two systems will be simultaneously used, it allows not only to have all the previous services as well as to add new services related to GSM. One of those new services would be to send the real position of the train.

The exact location of a train is of critical importance for safety as an integral part of a safe signaling system. New developments in navigation technology, such as those based on satellites, global positioning system (GPS), and especially differential GPS (DGPS), integrated with radio systems, have provided terrestrial structures with a good precision at a reasonable cost, making radio and satellite signals accessible.

That information is then sent to a centralized traffic control (CTC) that allows viewing, commanding, and managing all railway line circulation from a station. Train location, signal aspect, switch position, and the level crossing conditions, among other information, will be viewed through a projection system. The CTC shall allow the operators, with a global vision of the line, to respond in real time to all the situations that may occur. Through the CTC, one aims at achieving a better use of the existing resources, transport capacity increase, improved management of delays, better service quality, improvement of the links between trains or of trains with other transports, better schedule management, labor improvement, running costs reduction, reduction of time 
spent with conflict resolutions, and passenger information improvement.

Nowadays, train tracking and the guarantee of its integrity are currently based on the information received from the tracking systems (track circuits and axle counters) fitted along the railway.

Tracking circuits have been traditionally the usual way to detect trains, and it is a critical element for safety in railways [3]. Track circuits are systems used to track trains by using tracks as electrical conductors; they normally can track a train or a broken rail. The other well-known system is called axle counter [4] and is used for low precise train tracking. Its length from the transmission to the reception may vary up to a dozen kilometers. The axle counter system is a vital equipment for train detection, being an alternative to track circuit system. These are more useful in track section blocks, where there is a weak ballast, in tunnels, where the climate conditions are very humid, or as a redundant system in an area with track circuits.

Axel counter is based on the principles of absolute canton warning, namely, on the track section, considered to be occupied whenever any of the trains entered that section and may also be monitored when the train leaves that section.

Nevertheless, train detection is being developed using the train itself using the axle rotation as an integral part of the computer- and radio-aided train (CARAT) control system [4]. At present, the results of the tests on vehicles can establish its current status, namely, when running and on slopes. These assumptions are inherent to the counting method. Both of those systems have an infrastructure deployment time and cost extremely high for secondary lines, with train mild use, being impractical.

With the recent growth in satellite networks, the GPS system is also a viable solution to be used in train scenario. These GPS receivers combined with other systems (for instance, GSM) allow improvements in terms of communication velocity, offering the final consumer an increasing range of services. Therefore, in secondary railway lines which are not equipped with the tracking systems presented above (track circuits and axle counters), the GPS integrated with the GSM may rapidly grant the location and speed of a train to a centralized command station.

This low-cost infrastructure is thus viable for any secondary line, improving somehow the security of that track, and with the advantage of very low deployment time and work.

The main problem appears when we want to communicate and track trains inside a tunnel. The problem of locating trains in tunnels-and other locations where radio and satellite signals are blocked out or interfered with-is, however, a more complicated matter and is still a matter of research, while many other developments in other navigation fields can be adapted to the railway environment. Some systems, such as the overhead line inspection by vehicular equipment (OLIVE) [5], has already evaluated the use of GPS in railways. Nevertheless, since tunnels, barriers, and bridges can cut the line of sight to satellites, the integration of GPS with other sensors, such as gyroscopes, acellometers, etc., can improve this loss of GPS signal.

From [6], we can see that other technologies for train tracking include a microwave Doppler and optoelectronic sensors, which are nothing more than sensors based on track-to-train communications. This system is based on radio beacons located on the tracks with an approximate 10-m distance between them and transmitters and receivers on board of trains and in control stations, but the cost associated to develop this type of infrastructure is extremely high for use in low-budget lines.
Other projects are based on inductive cycles or on magnetic transponders, which track trains when they pass through beacons by changing the cycle phase or in a Doppler speed sensor which allows the location of trains by using the Doppler effect; that is, the wave frequency changes when the train moves in relation to the transmitter. The French railways combine the passive transponders, Doppler radar, and odometer to control the train movement in real time [7]. Other systems can also be used [6], but they all have the strong legacy of being extremely expensive and have a very strong deployment time.

That is why we propose in this paper an integrated system that simultaneously includes a radio communication system and a location solution. The proposed network will work as a redundant system where we have traditional radio and tracking systems, but it will also work on secondary lines where no other system is available. In fact, the overall system uses the train exclusively; thus, no terrestrial infrastructure is needed in secondary lines, cutting the deployment time and cost. Moreover, in the paper, we also present a low-budget system to track the trains inside tunnels, in this case and even if we need some form of repeating the GSM signals inside the tunnel, the location itself do not need any infrastructure deployment, since it will be based on the tachometer that exists in most of the circulating trains.

The paper will be organized as follows, first the track-to-train communications system will be presented, then the integration of the GSM and GPS modules [9]-[11] into this system will be described in Sections III and IV, respectively. This will be followed by the problem of GSM and location coverage inside tunnels and by proposing alternative schemes to it. Finally, some conclusions will be drawn.

\section{TRACK-TO-TRAIN RADIO COMMUNICATION SYSTEM}

In order to better understand railway communication systems, let us start by discussing the existing track-to-train radio communication system [1]. The track-to-train radio communications system is a central system, and therefore, any communication via telephony needs previous authorization from the regulator station (RS). Railway lines are divided, as far as regulation is concerned, in one or more sectors. Therefore, each sector of regulation is responsible for the regulation of a track section. The communications between interbase station (BS-equipment that makes the communication between the train and the regulator) and mobile station (MS - equipment included in the train which establishes the communication with the regulator) are carried out by means of a group of frequencies on the 450-MHz bandwidth, and each sector has three frequencies for emission and one for reception; that is, each BS emits in $f_{1}, f_{2}$, or $f_{3}$ and receives in $f_{4}$, and the MS transmits in $f_{4}$ and receives in $f_{1}, f_{2}$, or $f_{3}$ (Fig. 1). BS are set up so that the first BS transmits in $f_{1}$, the second in $f_{2}$, and the third one in $f_{3}$, and the following keep this frequency cycle, i.e., $f_{1}, f_{2}, f_{3}, f_{1}, f_{2}, f_{3}$, etc. This sequence is carried out so that the frequencies may be reused and to avoid interference among them; that is, when reusing $f_{1}$, the previous BS signal with the same frequency is minimum and is mixed up with the environmental noise and will not be considered by MS.

The MS will be recorded at the several BS along the line scanning the three frequencies and selecting the one receiving the highest level signal. There are four groups of frequencies allocated to Rede Ferroviária Nacional, EP (REFER-responsible for the Portuguese Railway Infrastructure) by the Portuguese Communications Authority. The existence of four groups of frequencies is due to the fact that railways in Portugal are interconnected at certain points of the country. A different group of frequencies is allocated to each line, so that those frequencies may be reused along the same line. At the interconnection 


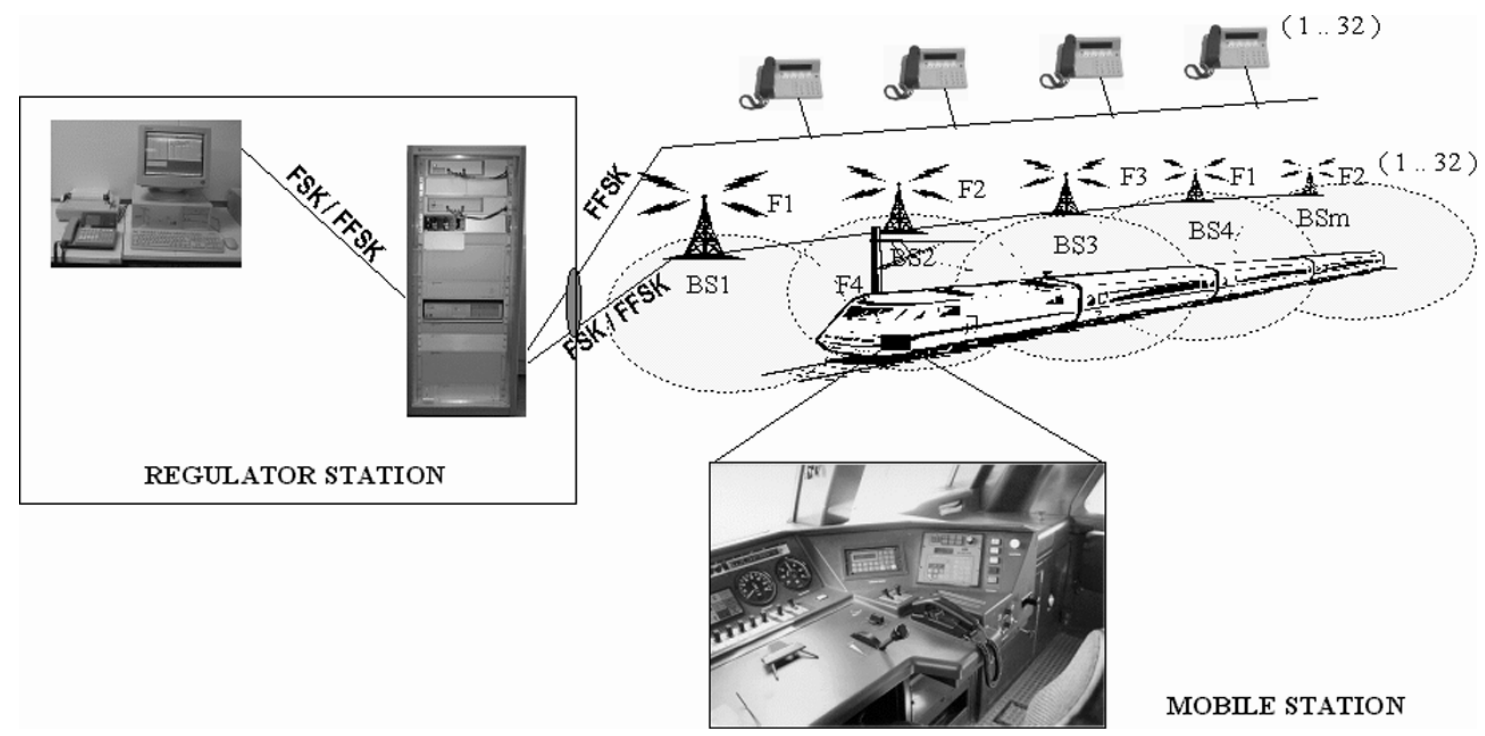

Fig. 1. Basic layout of a track-to-train radio network.

points with other lines, the chosen group is that of the main line (by main line, we refer the lines that are principal in the national railways, that means the lines that connect the principal cities, the industrial zones, ports, etc. The main lines will be in the future the main European railway corridors). The explanation, for the frequency group change, is related to the fact that if the interbase stations of the secondary line (secondary lines are the ones that are only used for lower traffic) had the same frequency group, the main line and secondary line signals would interfere with each other and the train would not be able to find out which interbase stations to register with. With the group frequency change, this problem no longer exists. Each regulation sector may only support up to 32 interbase stations due to the BS addressing. Several RS are installed at the CTC stations or local control stations. The function of the RS shall be to establish, control, and supervise all message traffic. The system comprises two base types of equipment:

- fixed equipment (RS, fixed station, and railway station);

- mobile, portable, and movable equipment.

In this system, there are three types of messages: telephony, status, and short data message (SDM).

The modulation used for the status messages is frequency shift keying (FSK) and fast frequency shift keying (FFSK). FSK subaudio at $50 \mathrm{Bd}$ messages are continuously transmitted by fixed stations during the conversations and signal to mobile stations the fixed stations status. These messages are transmitted in subaudio band in order not to disturb the telephony message exchange, and their transmission rate is low due to the modem type used, which does not allow greater speeds. FSK messages at $1200 \mathrm{~b} / \mathrm{s}$ are used for communications between fixed stations and RS via phone line (polling and traffic messages). FFSK messages at $1200 \mathrm{~b} / \mathrm{s}$ are used in selective calls and status service between MS and RS. SDM, modulated in FFSK at $1200 \mathrm{~b} / \mathrm{s}$, have appeared by the need of providing the MS with a data channel at $1200 \mathrm{~b} / \mathrm{s}$, in order to interconnect information boards, ticket machines, and new features such as frequency groups automatic changes, sleeping mode, etc., in the future. A SDM message may support up to 96 characters within its data field. There are three types of SDM messages: 1) Normal: in the event of a message coming in during a conversation between driver and regulator, the latter will go into a wait mode at the base until the conversation ends; 2) Urgent

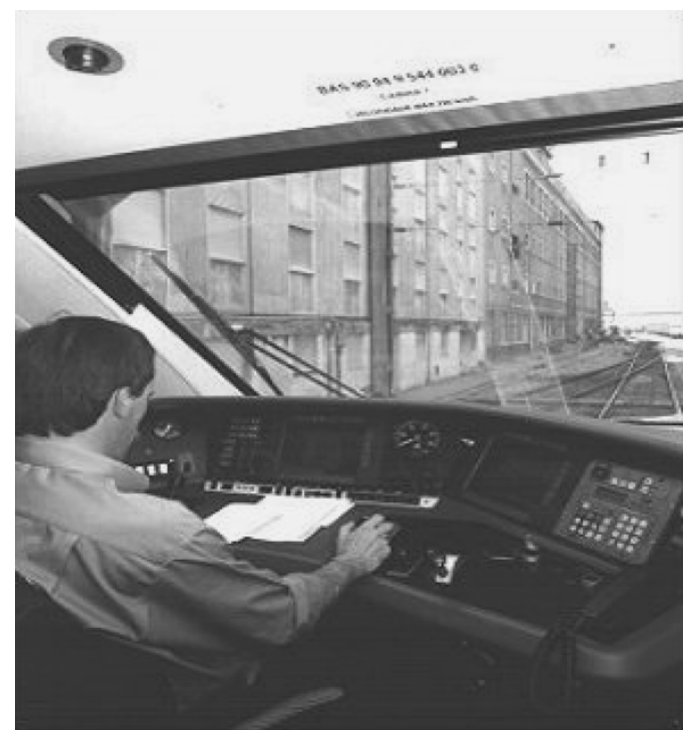

Fig. 2. Telephony desk (BG550 CP-N) and handset.

or high priority: during a conversation between driver and regulator, the latter will override the conversation; and 3) and Reply Messages: inform the REFER computer about the transmission result of a SDM, e.g., Mobile answers, Mobile does not answer, Mobile registered, Base station Busy, Base station does not exist, SDM not sent, SDM sent, Error alive message.

\section{A. Mobile Station}

Mobile station is the name of the equipment installed at the locomotives (Figs. 2 and 3) and that guarantees the communication via radio, between the train and the RS of the regulation section where the latter is registered.

All these connections have to be carried out through the RS. Communications between the MS and the RS may be carried out through telephony or status messages. An MS (Figs. 3 and 4) comprises a BG550 CP-N control unit which serves as an interface with the operator [man machine interface (MMI)], a handset, a loudspeaker, an antenna, and a Tx/Rx subrack, which in turn comprises a SE550 CP-N transceiver, 


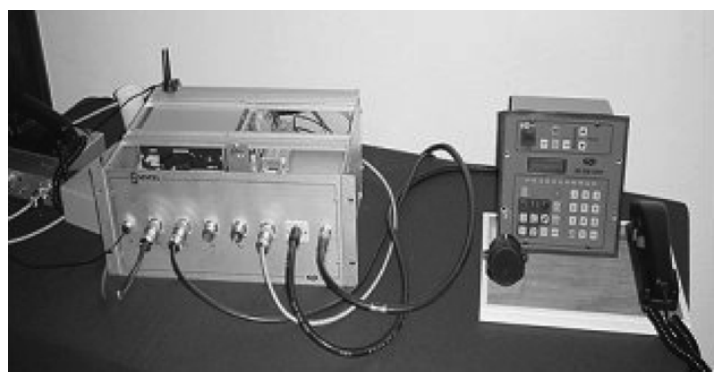

Fig. 3. Subrack, telephony desk (BG550 CP-N), handset, and loudspeaker.

which is a $\mathrm{T} x / \mathrm{Rx}$ transceiver for the radio frequency $(\mathrm{RF})$ communications, a data collection unit (ORD), a DC/DC converter, and one interface card with the passenger information system (AP).

The core of the whole system is an ORD universal interface that aims at the correct integration of different systems, translating protocols, data routing, or even equipment control. ORD carries out the interconnection between the transceiver and the mobile equipment being responsible for the interpretation and routing of the controls and data received by the transceiver. The ORD provides compatibility solutions between interfaces. Data obtained by different equipment items (telemetry, GPS, DGPS, etc.) may be collected by ORD (Device $1 \ldots 10)$ and routed to different communication equipment items (RSC, GSM, Tetra, Globalstar, Trunk, GSM-R, etc.). In short, its general characteristics are based on a Microcontroller M3771, with a Flash Memory of 512K, an SRAM of 32K, four analog inputs, four insulated digital inputs, four insulated digital outputs, and four RS-232 series interfaces.

The register unit belongs to the automatic train control system (RU-ATC) and records, among others, all the relevant data received from the ATC system evaluation unit through a current loop serial line. The recorded data is stored in a $512 \mathrm{kB}$ memory bank, enough for $12 \mathrm{~h}$ of data storage under normal driving conditions, beeing all the records time stamped and organized in five fields as first in first out (FIFO) registers:

- thumbwheels - with local ATC panel settings;

- events-with status of all ATC panel elements: keys, lamps, auxiliary, and main displays; six digital inputs; pressure order and emergency brake activation; traveling direction;

- continuous - speed, distance, tachometer pulses, and main pipe pressure drop, recorded every second in normal operation or every $30 \mathrm{~s}$ when at stop;

- beacons-wayside information (maximum five beacons with three words each);

- special-messages sent by the RU-ATC to the radio system (track-to-train communications system).

The RU-ATC drives the ATC speedometer with the values received from the ATC, or with its own calculations from a three-phase optical tachometer sensor when the ATC is switched off (according to internal $\mathrm{CP}-$ Portuguese Railways rules, the RU-ATC is always powered up). One relay output is available to give external alarm. One RS232 port provides access for configuration and data read-out by a standard PC. During normal operation, this port is permanently connected to the vehicle radio system (track-to-train communications system) in order to send relevant messages such as ATC status (ON or OFF), beacon error, emergency brake, entering/leaving station (special beacons), RU-ATC alarm.

\section{INTEGRATION OF THE GSM COMMUNICATION SYSTEM}

As was previously discussed in Section I, the GSM integration aims at solving the lack of communication between local control stations/centralized traffic centers with MS, in lines where no track-to-train radio coverage exists, which means mainly on secondary lines where the development of a complete core communication system is extremely expensive. Thus, came the idea of integrating GSM in track-to-train radio, using one of the existing GSM network operators (Fig. 5) [12].

With this integration, the data transmission goes from 1200 to 9600 $\mathrm{b} / \mathrm{s}$. This alteration is carried out at the level of the rack components of the MS and at the time of the change due to a dual-mode antenna. In this case, the same interphone desk may be used and it is not necessary to make new drillings or cable runs for this purpose. In short, this development is aimed:

- to provide MS the access to GSM public network, enabling direct interphone communication, between traction permanent and the driver;

- to increase the data transmission capacity (SMS) to a transmission rate from 1200 to $9600 \mathrm{~b} / \mathrm{s}$.

As shown in Fig. 5, the GSM interphone works independently from the physical track-to-train radio network, but if during a GSM call, the regulator needs to communicate with the driver, this GSM call goes into a wait mode and will only be reconnected when the train-to-track radio connection is ended; that is, train-to-track prevails against GSM when it is present. This additional data transmission at $9600 \mathrm{~b} / \mathrm{s}$ may be used at the Depot Management, which has some advantages over the solution foreseen for the train-to-track radio such as it is infrastructure-free and the speed rate is eight times greater. To implement these new features, it is necessary to reconfigure the hardware and the algorithm of the MS, which changes from what is given in Fig. 6 to Fig. 7.

The SE550 radio equipment, the optical link interface (ILO), the RU-ATC, and the Octopuce hardware (Interface Universelle Octopuce, developed by the Octuor Systeme in France, is the processing core of the overall system) were not changed. Only the SE550-ILO block had to be redesigned; this block has mainly two functions: the selection of the audio communication channel and the control of the GSM modem. The RS-232 coming from the SE550 and ILO interface was transferred to the Octopuce interface. A low-frequency audio switch was placed on this card, enabling the connection of the low-frequency audio between the ILO interface and the SE550, or between the ILO interface and the GSM. At the default position, the low-frequency audio is connected between the SE550 and the ILO interface (original assembly).

The ORD shall establish the timing for the low-frequency switch. With this new assembly, the ORD requires six serial ports (Figs. 8 and 9). The SE550, the future applications computer, and the RU-ATC have the same original assembly. The COM4 shall be appointed to GSM module (Fig. 9). To replace the two missing ORD serial ports, a daughter card has been added. The daughter card of the Octopuce interface is provided with two extra serial ports. These ports are connected in SE550 and ILO. With the new MS diagram of Fig. 7, the ORD system should intercept a BG550 CP-N from cabin 1 (BG1) or a BG550 $\mathrm{CP}-\mathrm{N}$ from cabin 2 (BG2) command to be able to use the GSM. For a transition between SE550 radio and ILO, the ILO command received by ORD shall be generated in SE550 serial port. Therefore, during a telephone call, the low-frequency audio signal shall be shifted to radio. The ORD establishes the activity between SE550 and ILO messages in the serial port. This surveillance allows for a correct managing of a 


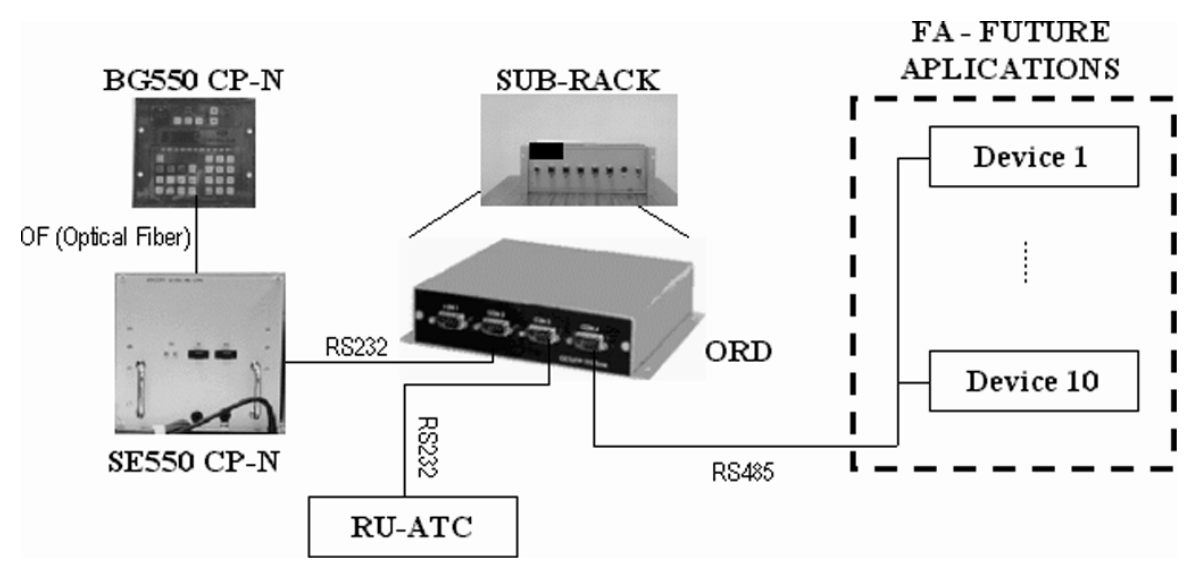

Fig. 4. Mobile station diagram.

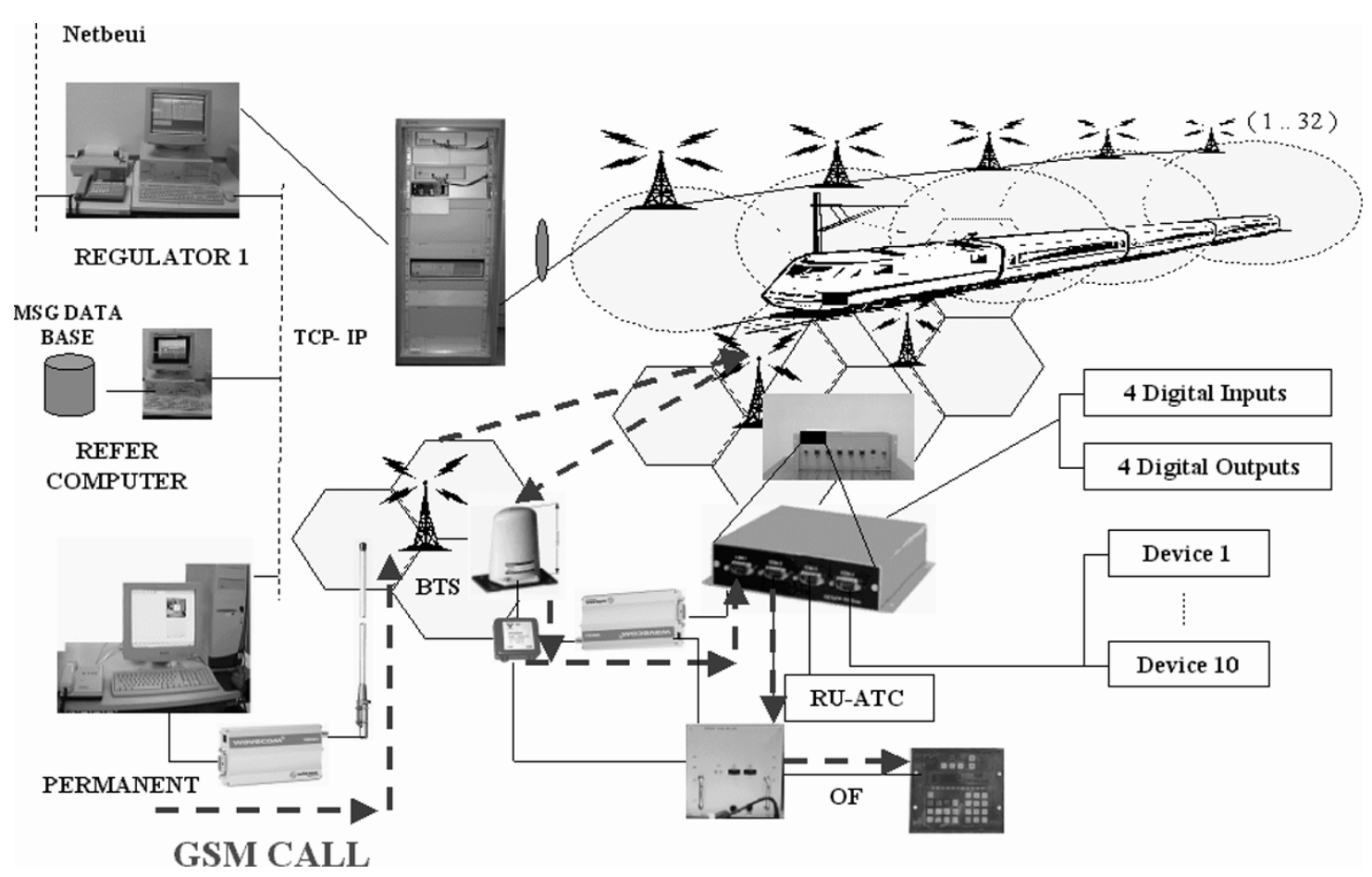

Fig. 5. Telephony via GSM working scheme.

possible conflict arising between various future calls. In a critical case, the ORD shall be ready to refuse the next GSM calls.

To avoid the modification of the RU-ATC system, some status messages may be sent through GSM. The ORD shall establish the radio support for the transition status (according to the status number). The GSM modem is connected to Octopuce through COM4 port. In short, to extend the track-to-train network for the GSM, it was necessary to develop an extension of the ORD algorithm, to place a daughter card in the ORD, to install a GSM module, and to redesign the SE550-ILO card. However, the connections to the interphone and passenger information system (AP) were not changed and the telephony communications through GSM network are in half-duplex due to the used BG550 $\mathrm{CP}-\mathrm{N}$ and handset.

In a telephony call, the MMI of the permanent (desk) PC allows placing a voice call to a train, using GSM or public switched telephone network (PSTN). The MMI shall also signal calls received. The features that were implemented are mainly call reception signaling, subscriber number of the caller visualization, connected calls, short dialing management, specific features of the system.

It should be noted that for safety reasons, the reception of an ALARM interrupts the GSM call, and in the case of a regulator call coming in the middle of a GSM call, the GSM call will be kept in standby for a customized period, at the end of which the call will be ended; however, if the regulator-driver conversation end during the standby period, the GSM call shall be resumed. When the MS is involved in a GSM call and an RS call comes in, instead of keeping the call on wait, it is possible to end the GSM call $5 \mathrm{~s}$ later. As a result, the MS is not charged on account of the waiting time. When the MS is involved in an RSC call with the RS and a GSM call arrives, the GSM modem of the MS should reply with the busy signal tone. It is possible to send and receive STATUS and SDM through the RSC system during the GSM call. For communicating with the central station, there 


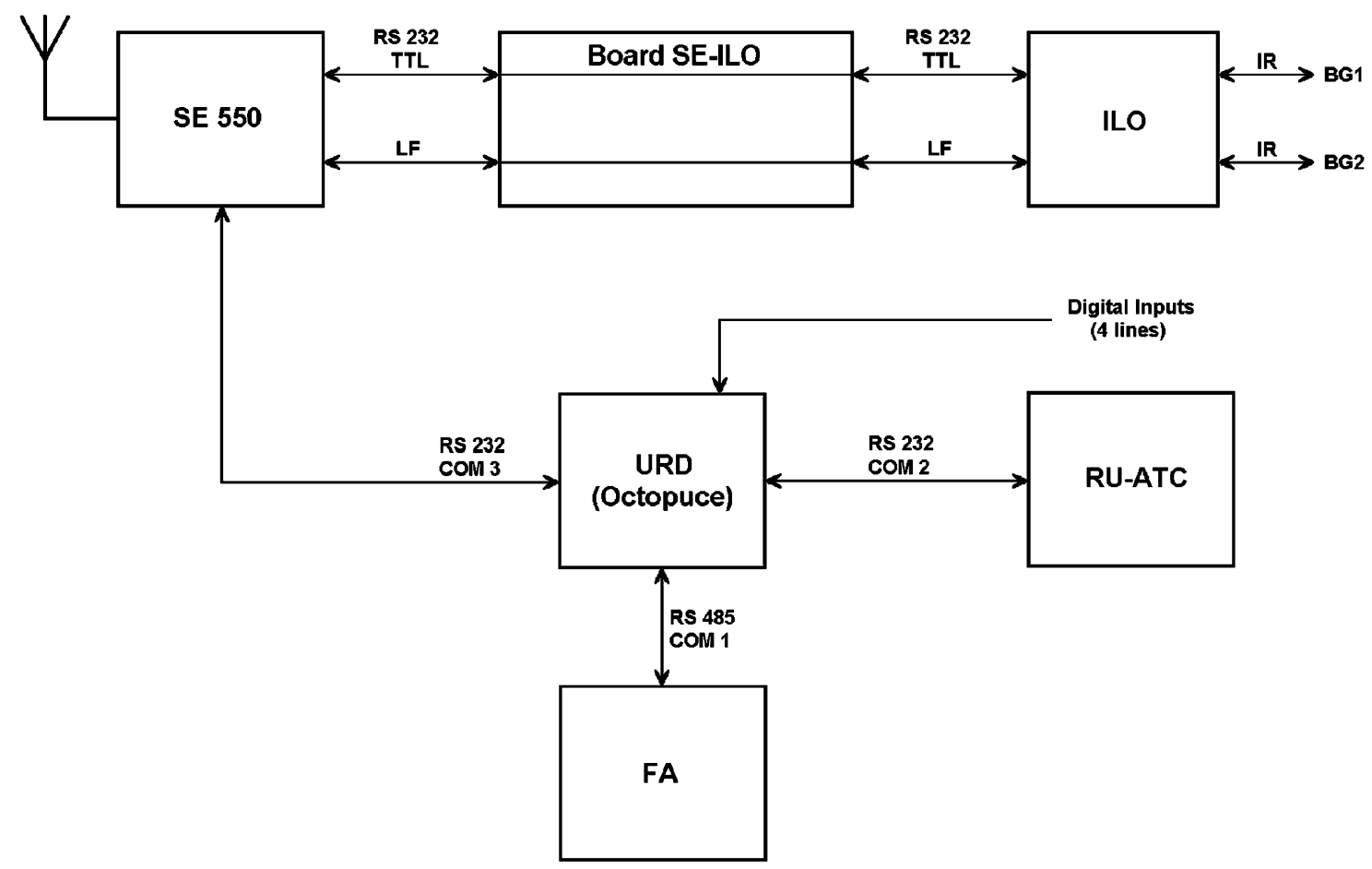

Fig. 6. Diagram of the mobile station of the track-to-train radio without GSM.

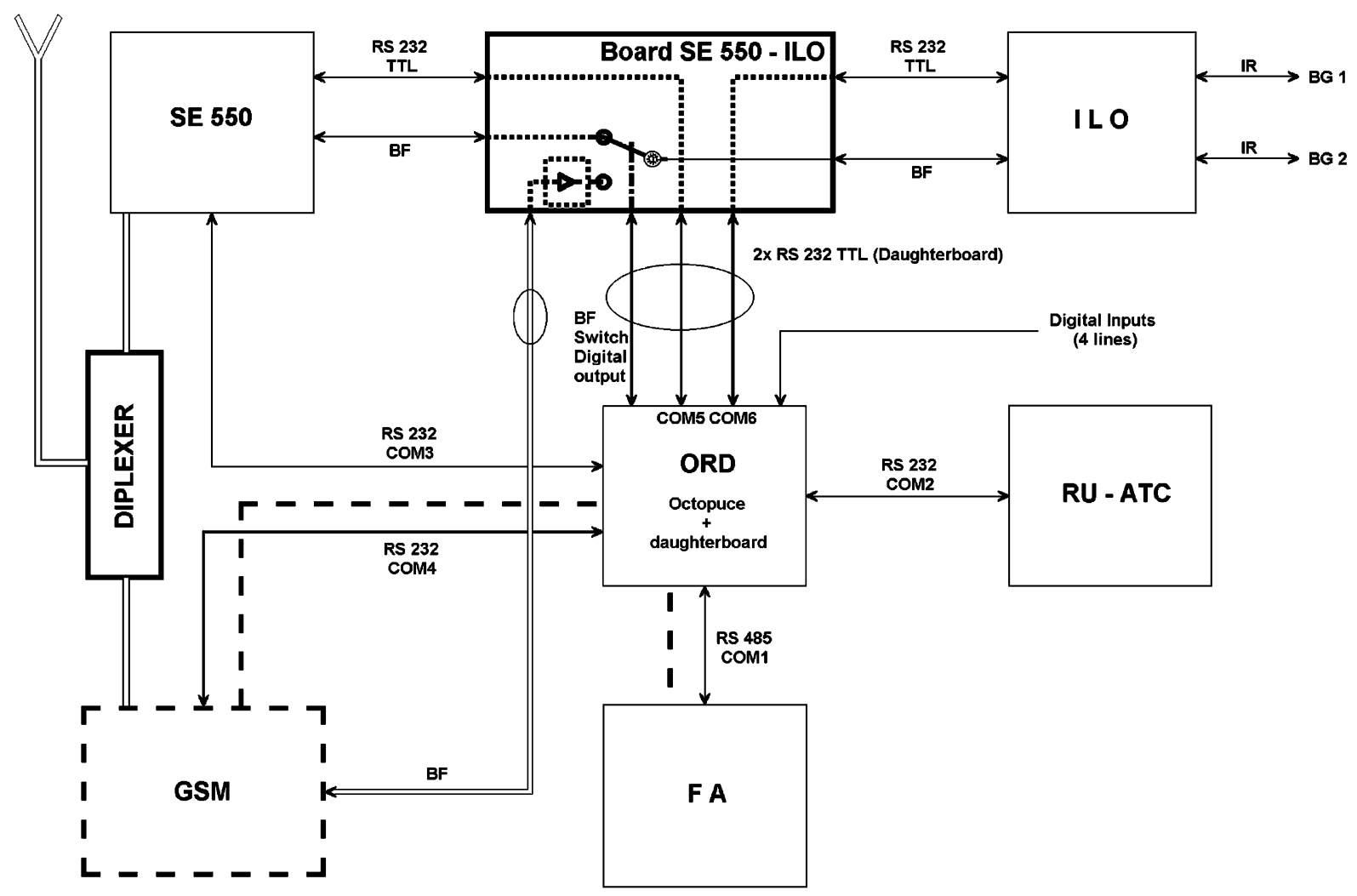

Fig. 7. Diagram of the mobile station of the track-to-train radio with GSM.

are three possibilities for data transmission between the permanent traction and one of the ten users of the MS RS-485 open bus:

- SDM via RSC;

- SDM via GSM;
- data transmission via GSM in free format and transparent mode at $9600 \mathrm{~b} / \mathrm{s}$.

The SDM is a free format message carrier between the embarked equipment and the RS. Once the data connection is established, we can 


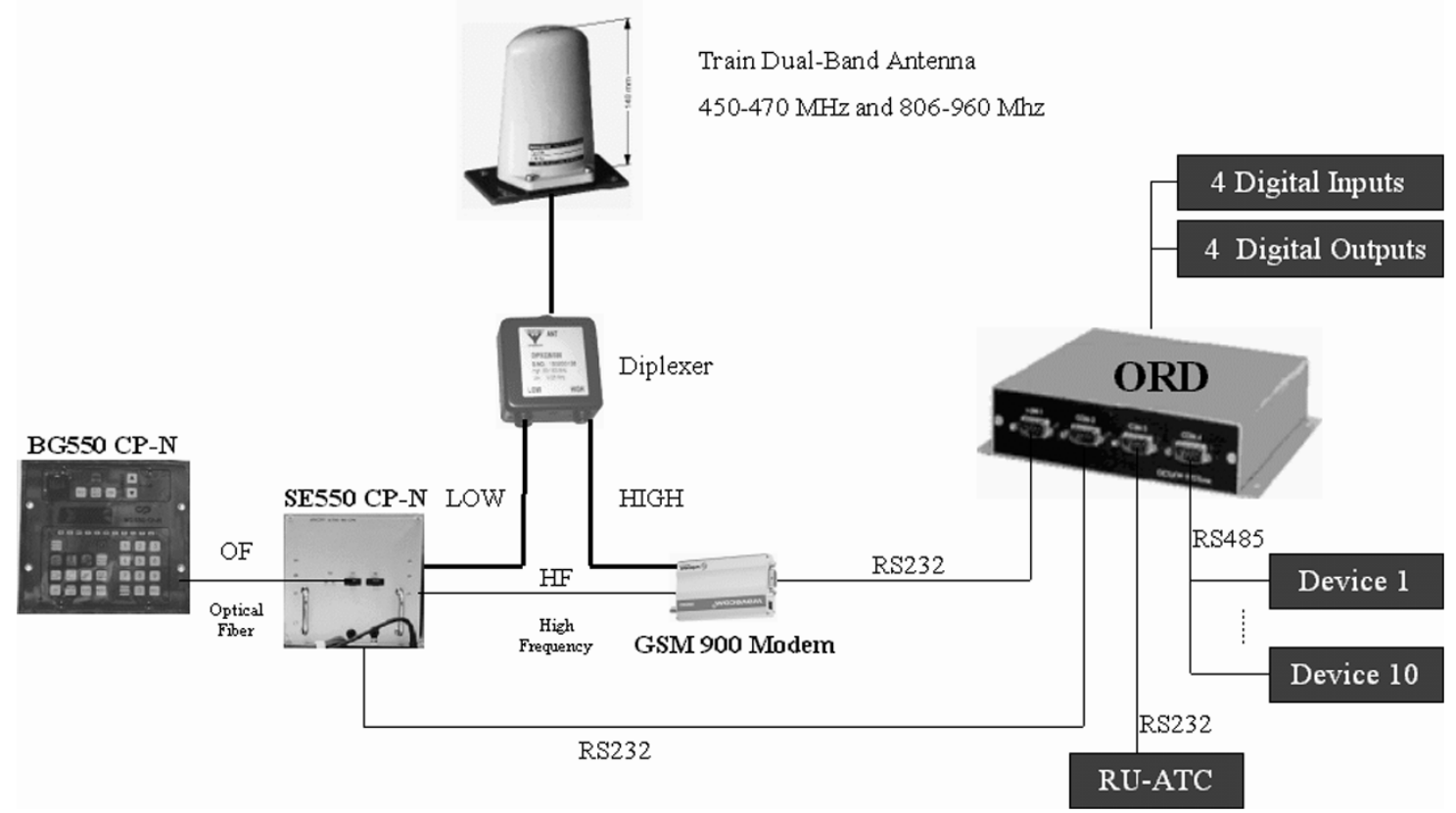

Fig. 8. Mobile station with GSM.

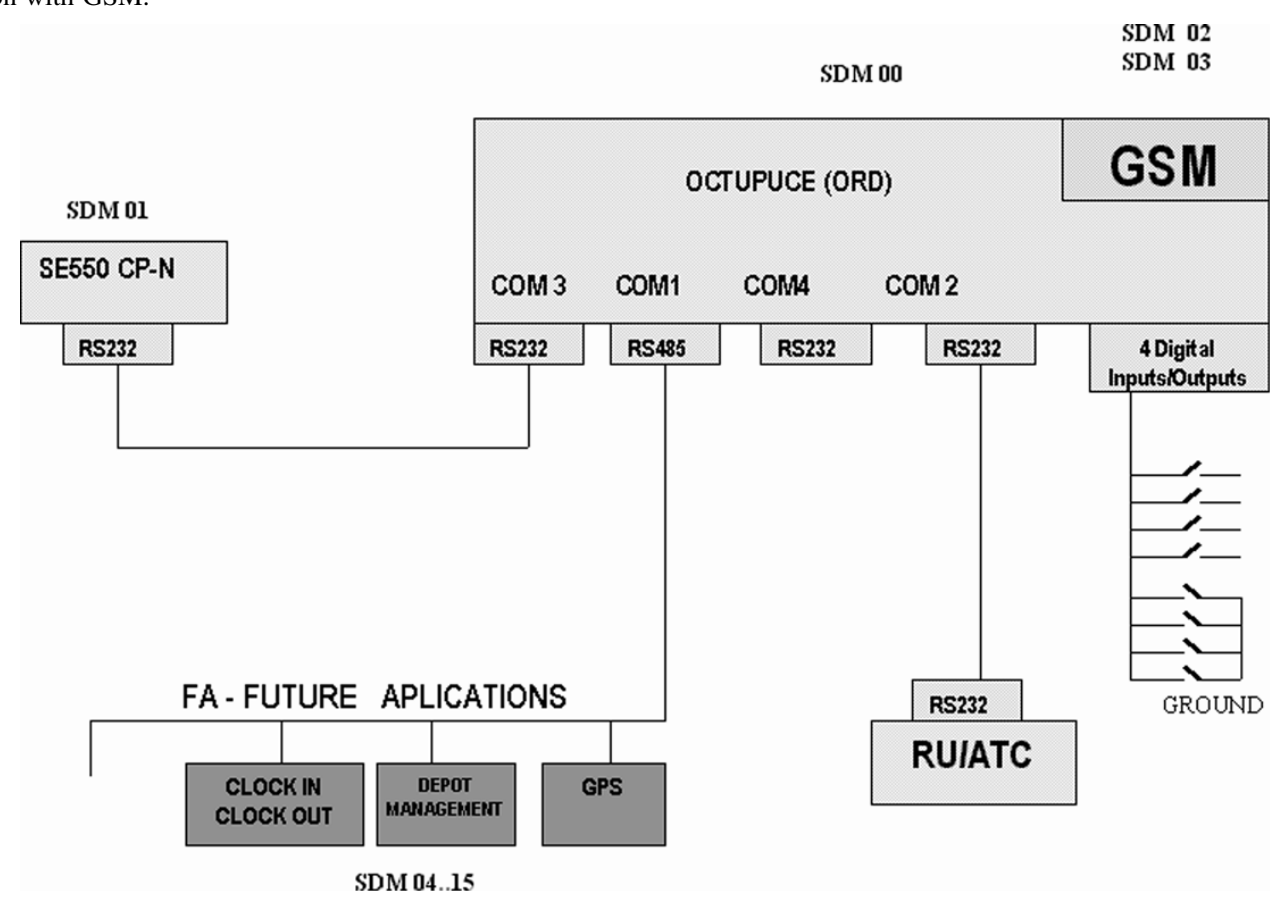

Fig. 9. Data collection unit with GSM.

consider that the permanent PC and the future aplications (FA) are logically connected. Z-modem, X-modem, Kermit, etc., transfer protocols may be used. Fig. 10 presents the overall system with GSM integration.

It is interesting to see that the GSM integration allows the inclusion of a redundant system into the track-to-train available security communication channel, but it allows trains traveling in secondary lines to have some form of communication signals with the station.

\section{INTEGRATION OF THE GPS CAPABILITY}

Having in view one further track-to-train radio development and bearing in mind the previously mentioned tracking needs of the railway operators, mainly on railways not yet provided with the abovementioned tracking methods (track circuits and axle counters), the GPS system integration on track-to-train radio system is a successful alternative (Fig. 11).

In order to integrate the GPS module in the communication system, the ORD universal interface aims at the correct integration of the different systems, translating protocols, routing data, or even controlling the equipment items. The ORD, as the core of the integrated system, shall have the task to centralize and route all information exchange, coordinating all the equipment items and translating information between them, Figs. 12 and 13 present the overall system.

The expanded interface with integrated GPS allows for the geographic location of the mobile, and the radio interconnection is ensured by the proposed interfaces. A flash memory enables keeping the data record for long periods of time. The serial ports are TTL 


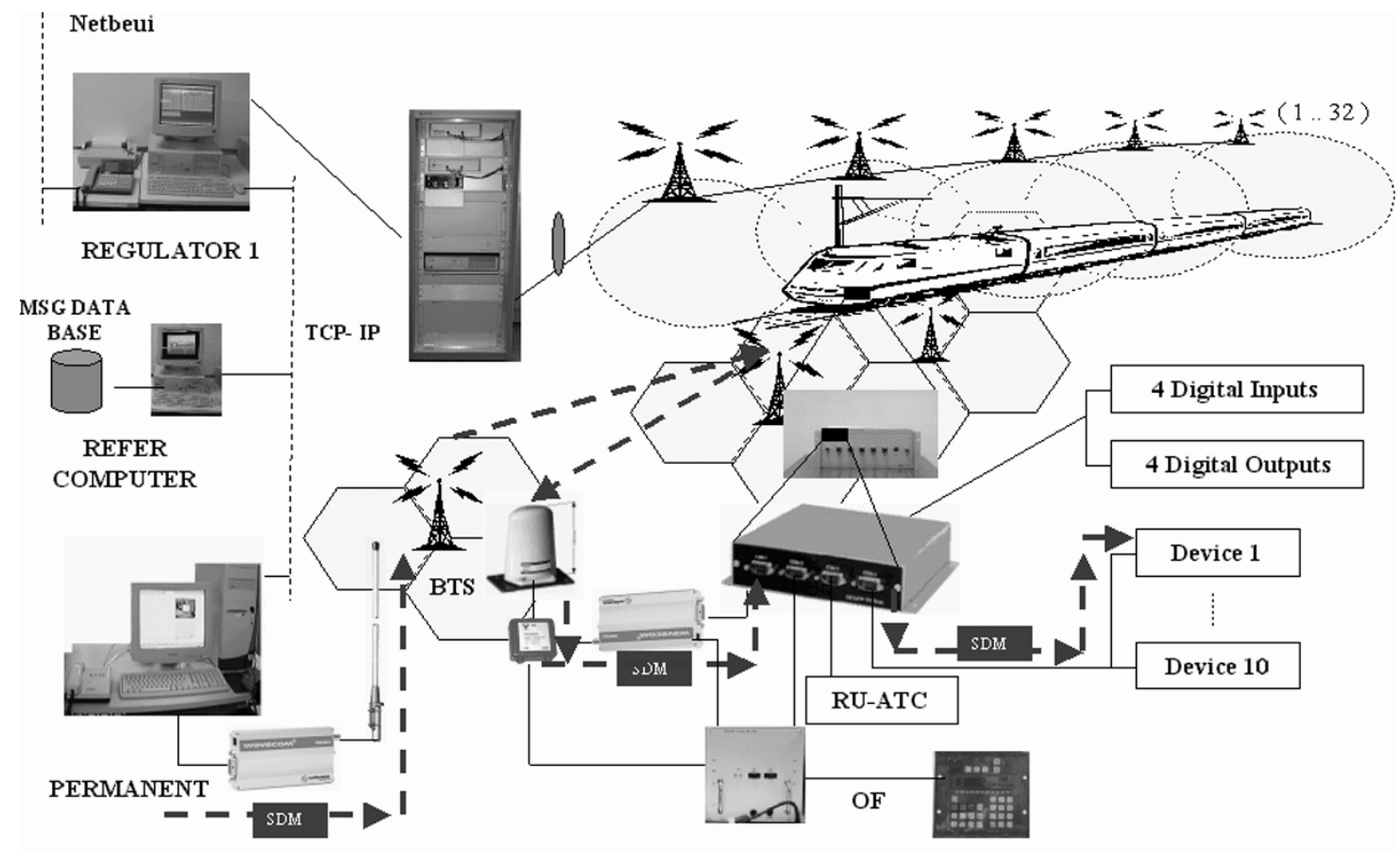

FILE DATA

Fig. 10. Data transmission via GSM.

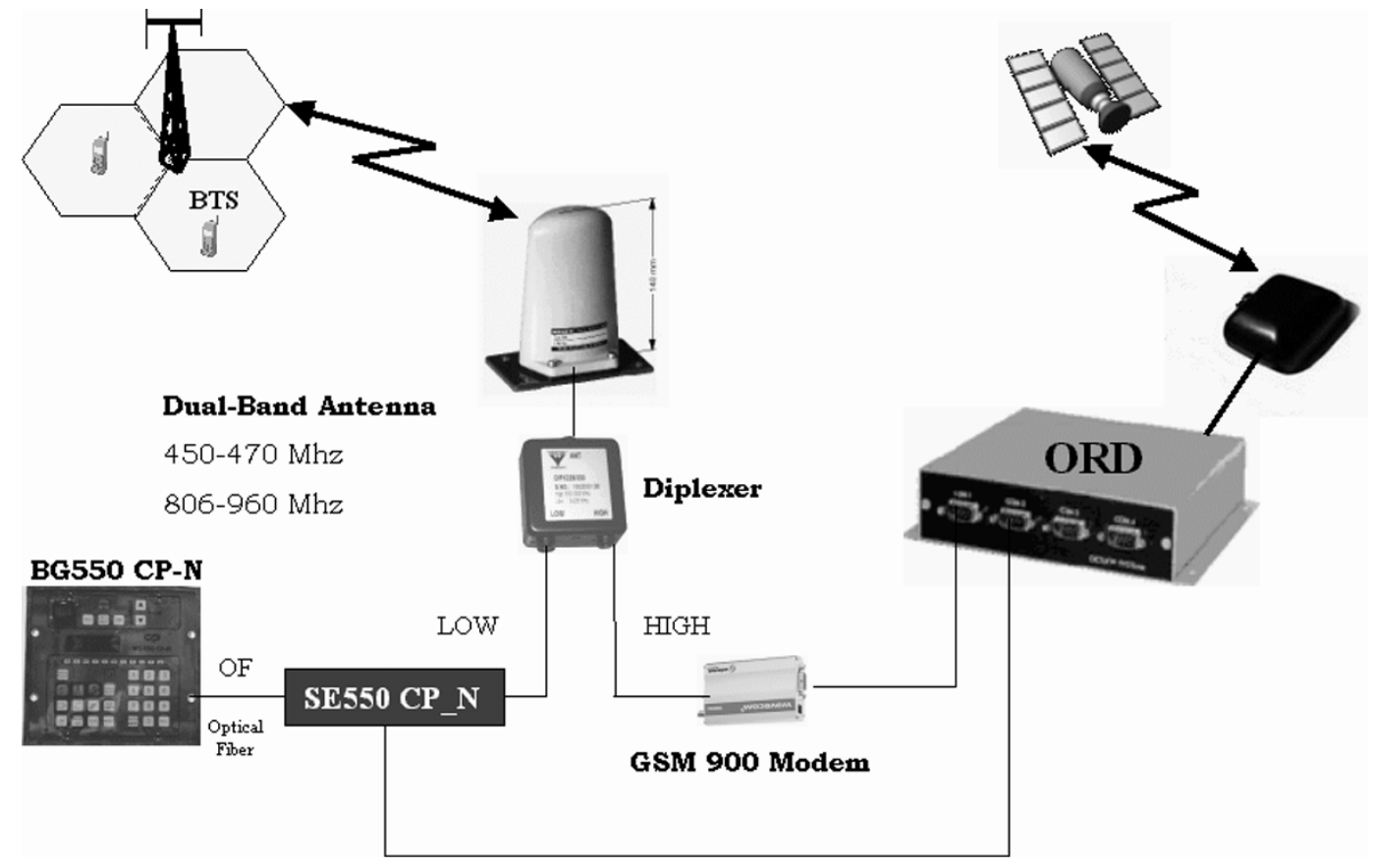

Fig. 11. Track-to-train radio with GSM/GPS.

levels RS-232 interface, and COM5 and the COM6 may be used to carry out this operation. The COM5 serial port allows for the transfer of controls and positions, while the COM6 serial port allows for the correction of the position by using a DGPS receiver (differential mode). GPS provides for a pulse per second (PPS) periodic signal of 1-s satellite synchronized. That signal may enable, for instance, the transmission synchronization coming from several other interfaces. The DGPS mode (differential mode) needs two serial connections because a correction frame should be supplied to the GPS so that it can correct the position. That correction frame may be transmitted by a COM6 or originate on the 25-pin connector [from Radio Technical Commission for Maritime Services (RTCM)].

The whole system shall be based on the connection diagrams illustrated in Figs. 14 and 15 for fixed and mobile stations. This means that it will be necessary to configure the two ORD to allow for clock configuration and all the protocol parameters required for the communication of the former with the GPS system (GSM phone numbers, communication speed, number of bits, parity type, and the serial ports to be used). After inserting all these data, they will be downloaded into the ORD. After the installation of the several programs required for the good op- 


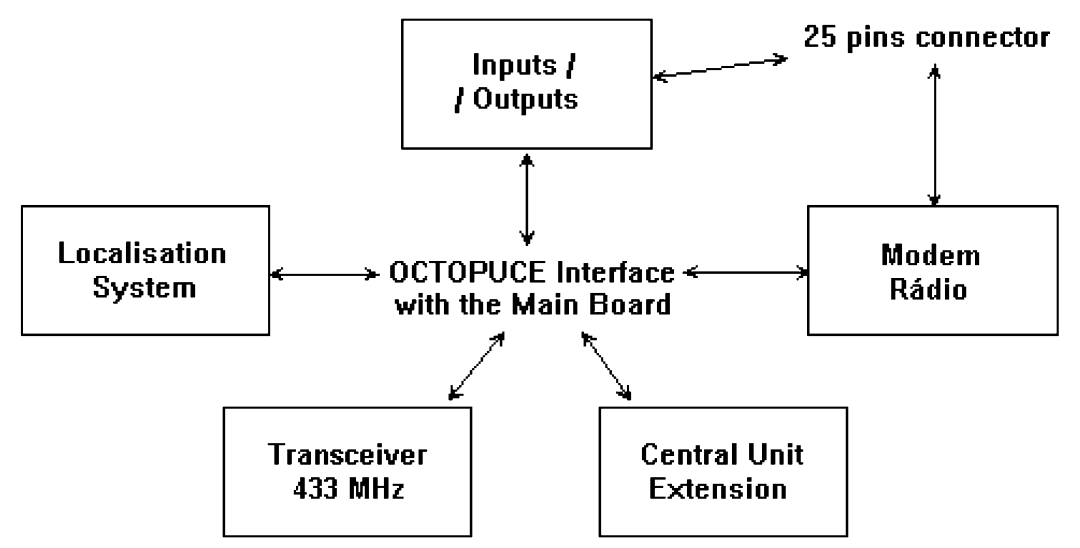

Fig. 12. General scheme.

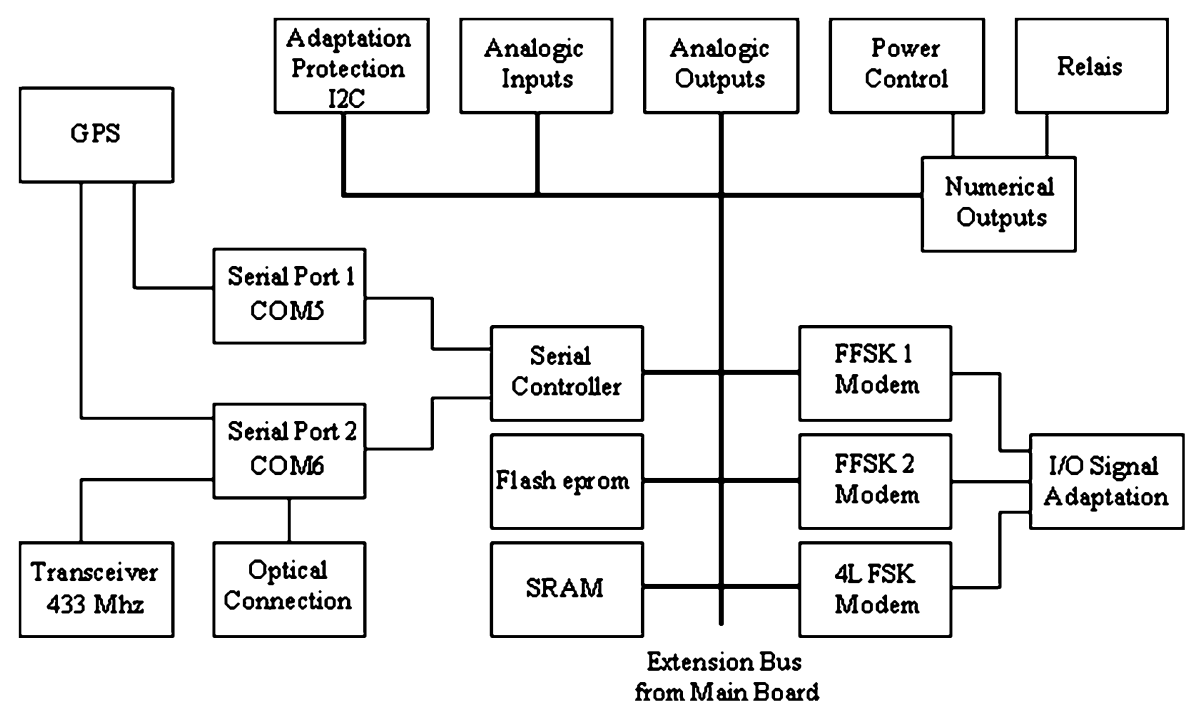

Fig. 13. Functional diagram.

eration of the location system via GPS, it is then necessary to run its applications and carry out the respective connections, both in the fixed and mobile stations. The positioning results are then packed accordingly with the NME-0183 protocol.

\section{TRACK-TO-TRAIN COMMUNiCATIONS AND TRACKING IN TUNNELS}

Because when we lose line of sight to satellite (GPS), e.g., in tunnels, we cannot locate the train itself; this problem should therefore be carefully studied. Not only the communication channel disappears, but the GPS signal is also of no use.

Thus, for this system to continue to work properly, it should be possible to allow GSM signals to be transmitted inside tunnels as well as to provide an alternative to the GPS location system. To do so, GPS alternatives should be available [6], [8], and we should have communication paths inside those tunnels. Precisely the reason we first evaluate the GSM coverage of tunnels and then the integration of other sensors with the GPS receiver.

\section{A. GSM Coverage Inside Tunnels}

For the information of the location of a train inside a tunnel to be sent to an RS, there has to be GSM coverage inside. Therefore, it is necessary to provide a tunnel with adequate equipment to allow its coverage. Besides, the communication systems in tunnels need to be used not only by railway agents but also by emergency services. This implies that the radio communication system to be installed in tunnels will have to be similar and have to bear the same requirements as the emergency services. Next, we present different types of solutions for a GSM coverage in tunnels.

The solutions spans from single repeaters to the use of an agglomerate of repeaters, with or without redundancy. If the tunnel is small in length, only one exterior repeater is normally enough and should be placed in one of the tunnel's entrances, as shown in Fig. 16.

In case there is a need for redundancy, two separate repeaters should be placed in each entrance and their signals will be sent to two different base transceiver stations (BTS; Fig. 17), which should be placed near the tunnel. For a better coverage, we suggest a combined solution of a repeater connected by fiber optic and signals sent through a leaky feeder.

Due to an optimum planning of the network, the BTS may not be near the opening of the tunnel, and hence, Fig. 18 suggests an alternative to assure a radio coverage within a larger tunnel. Where there is a need for redundancy, there should be two repeaters connected to two different BTS through optical fiber.

When the BTS is located near a tunnel, a "divider" will divide the aerial signal inside the tunnel, covering the first part of the tunnel. An optical HUB, through fiber, will link the local repeaters inside the tunnel, thus covering the remaining of its length (Fig. 19). 


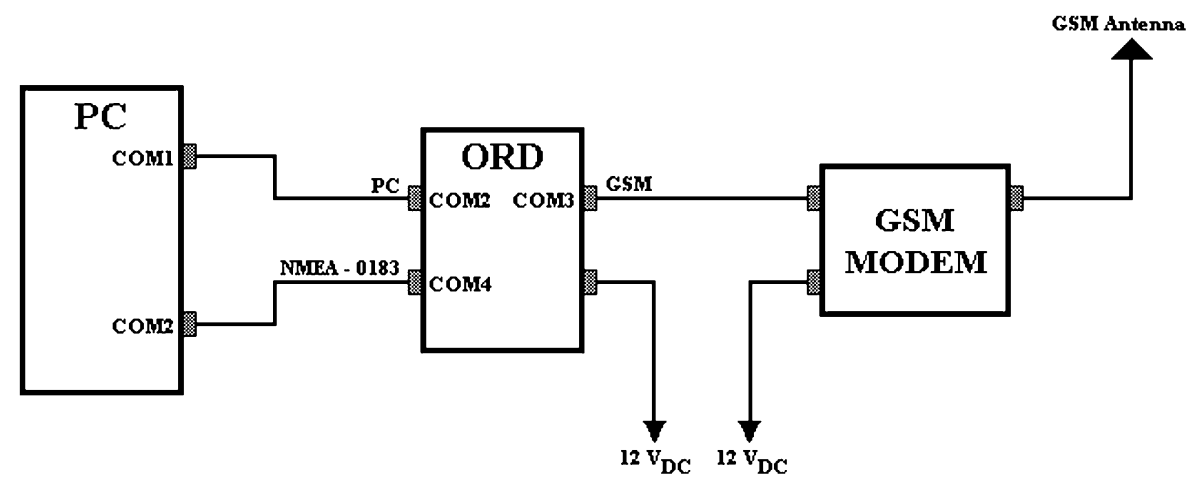

Fig. 14. Connections diagram of fixed station.

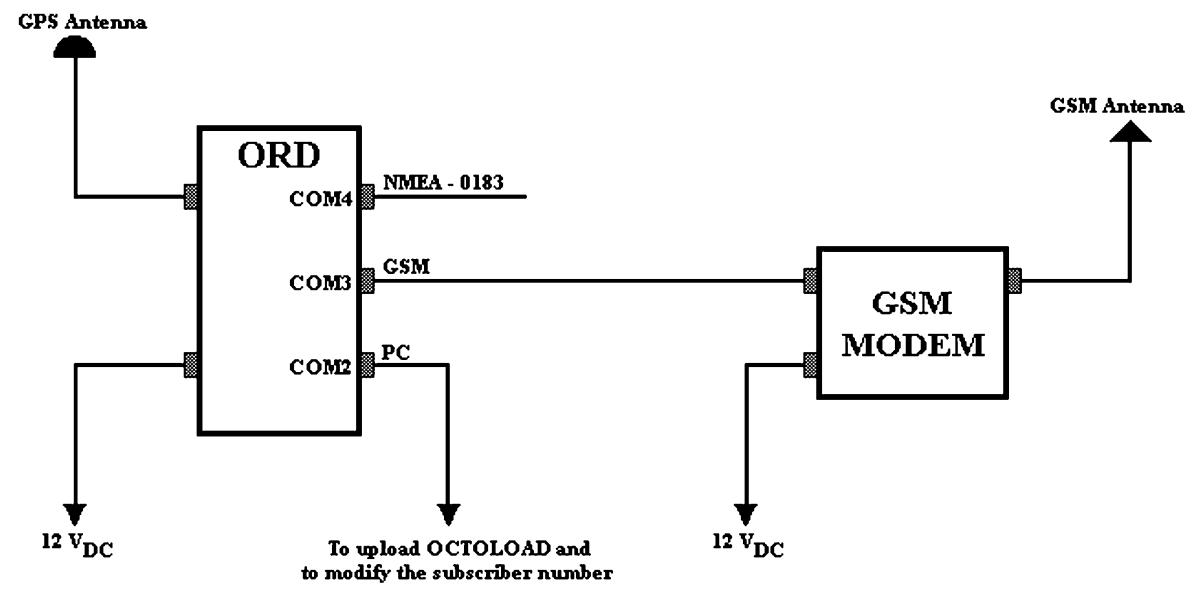

Fig. 15. Connections diagram of mobile station.

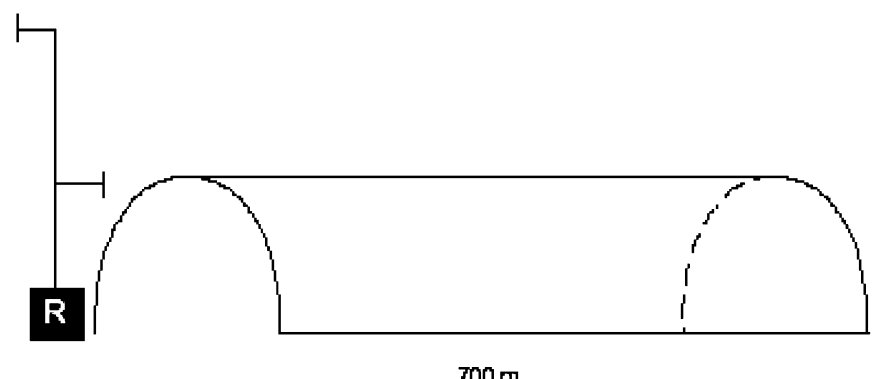

$700 \mathrm{~m}$

Fig. 16. Exterior repeater located at the entrance of the tunnel.

When redundancy is demanded for safety reasons, two BTS are connected to the tunnel's repeater system, as shown in Fig. 20. In this case, we have two basic systems that guarantee double coverage. This way it is possible to have GSM coverage inside the tunnels and thus continue to feed the central station with location information.

One practical example of RF coverage inside tunnels, already implemented, is the 12,750-m-long Peloritani Railway tunnel project in Sicily, where the Italian Railways decided to adapt the tunnel to today's railway traffic needs. To enhance radio coverage, a fiber-optic system was installed. Head stations have been installed on each side of the tunnel. Each head station interface itself with the outdoor BTS processing, converting RF signal into optical signals. Optical signals are propagated via single-mode fiber-optic distribution to optical repeaters where they are converted into RF signals then amplified to enhance coverage. Each head station is connected to six optical repeaters; about at the half part of the tunnel, handover between BTS can be performed.

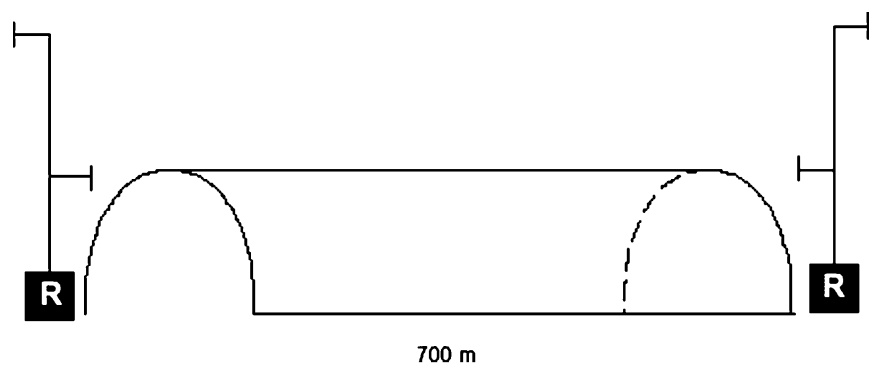

Fig. 17. Two exterior repeaters placed on the tunnel entrances.

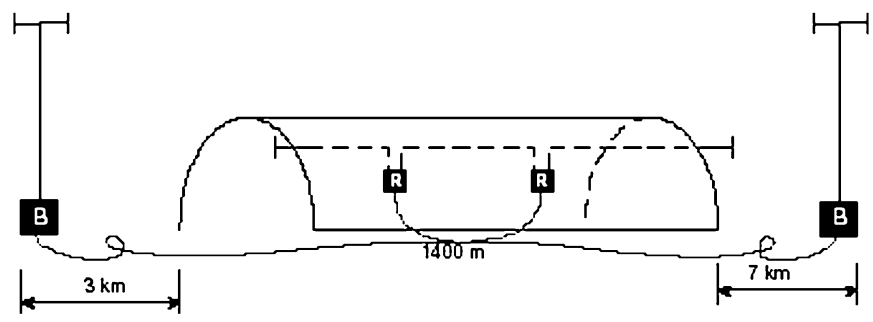

Fig. 18. GSM coverage with redundancy.

\section{B. Alternative to GPS Inside Tunnels}

Since tunnels in a railway network are mostly of short length (namely, in Portugal, where tunnels are $500 \mathrm{~m}$ long, or less), they are, in addition, straight. Knowing that all running trains are equipped with tachometers and/or tachographs, we may integrate the signals from this equipment in the track-to-train communications system; through quick 


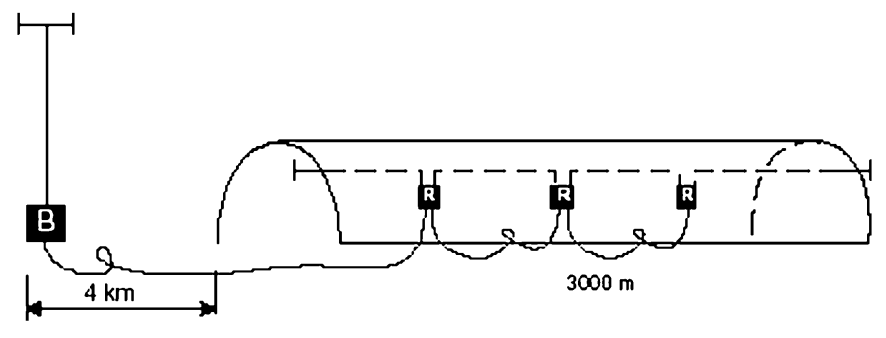

Fig. 19. Coverage in long-length tunnels.

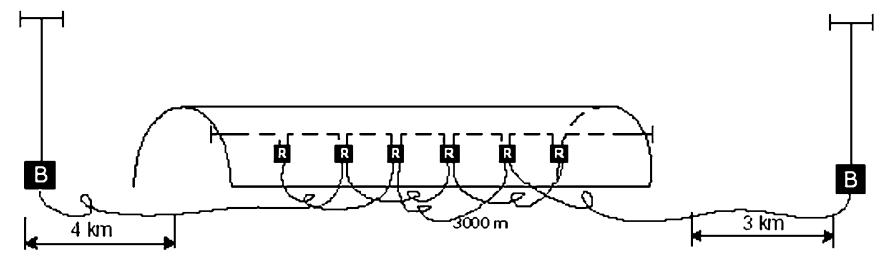

Fig. 20. Coverage in long-length tunnels, with redundancy.

processing at the ORD algorithm level, we may continue knowing the location of the train when the GPS signal is absent and send it to a railway and traffic control management center through GSM. This solution is better than using an odometer accelerometer and/or gyroscopes, which must be installed on the trains, as tachometers are already available. Consequently, we would have the MS block diagram shown in Fig. 21. The algorithm to be developed for data processing from tachometers/tachographs must contain the basic principles given in Table I.

As one may verify, whenever there is a GPS signal, that coordinate will be sent via GSM. Otherwise, the ORD processes the data from the tachometer/tachograph jointly with the last coordinate received by the GPS and sends the resulting coordinate through GSM. For such, it is necessary that the ORD algorithm keeps in memory the last resulting coordinate sent by GSM, for the need of a future processing, as well as the last sense of direction (course) received via GPS. When a train is located and moving in a tunnel, it should immediately start counting the number of impulses from the tachometer/tachograph $N$, having the wheel diameter $D$ already been preprogrammed, we can know the distance traveled $d$ within a period of time, through:

$$
d=\frac{\pi D N}{n}
$$

where $n$ is the number of impulses per rotation sent by the tachometer/tachograph. Subsequently, through the last sense of direction $\theta$ sent by GPS and kept in memory, we may know what the mobile's Cartesian displacement was (Fig. 22).

Subsequently, through a simple calculation, we know what the new latitude and longitude coordinates of the mobile were.

$$
\begin{aligned}
\text { LATITUDE }_{\mathrm{NEW}} & =\text { LATITUDE }_{\mathrm{OLD}}+\Delta_{\mathrm{LAT}} \\
\mathrm{LONGITUDE}_{\mathrm{NEW}} & =\mathrm{LONGITUDE}_{\mathrm{OLD}}+\Delta_{\mathrm{LONG}}
\end{aligned}
$$

Last, the latter coordinates are sent through GSM for the respective control and management center. One should consider that this proposal should only be applied in places where one may generically consider that a railway tunnel is relatively "straight," and thus one may use the capacity existing in the ORD of the track-to-train communications system and of the tachometer/tachograph within any train. Consequently, several calculations were made to have a real notion of what the mistakes presented by this proposal are in case it was chosen and in case the tunnels were not straight. It is worth mentioning that in Table II it was considered that a train runs short distances in minor curvature radiuses and runs longer distances in larger curvature radiuses.

We may conclude that the solution presented is simple and of low cost, when compared to solutions with resource to beacons, direct holes in tunnels to receive GPS signal, for the interaction with other previously described systems or with the integration of a gyroscope. At worst, precision errors stand in the range of hundreds of meters, which may be more or less critical, depending on its final use and of the quantity of local traffic. Knowing that in order to run at higher speeds, trains need larger-curvature radiuses, thus verifying that the precision error is strongly diminished, being comparable to the GPS precision errors. Besides, this solution is much more immune to electromagnetic interferences than the other systems described. Integrated solutions of the abovementioned several sensors require verifications of consistency and the use of analytical redundancy techniques in case of signals from sensors which are broken or seem unreal. The abovementioned technologies or an integrated solution will have to be assessed in terms of their comparative performance, i.e., precision, confidence, maintenance, considerations of involvement in the railway area (tunnels), aspects related to safety, and cost for the several types of railways. Trains may be measured in different classes. In each one of those classes, a number of items may be considered. The needs for location of the several types of trains and other mass traffic systems are clearly different. Mass traffic systems are quick, and in short distances, they may need information of their more exact location than freight trains, which are slower, keep more distance between them, and run longer distances. The several needs are also very dependent on the application of the location. For example, test vehicles used by the infrastructure, monitoring of the track, and maintenance activities may need a more sophisticated location equipment to be faced with other parameters that are being locally registered, but may not need the same strict safety verification as for train/signaling control applications.

\section{FUTURE IMPROVEMENTS}

Access to mobile Internet and telematic services are one step away from the general public, such as vehicle identification, freight information, and other services using GSM data services [13]. Needs of two types may be clearly identified by the market. Many applications, such as telematic services or many railway applications, have low data transmission rates and have a typical burst transmission. Starting with a 14.4 $\mathrm{kb} / \mathrm{s}$ data transmission rate, it will be increased with data circuit-commuting technology. The high-speed circuit switched data (HSCSD) will use channel aggregation to allow a high data transmission. Starting with $28.8 \mathrm{~kb} / \mathrm{s}, 64 \mathrm{~kb} / \mathrm{s}$ transmission rates, such as the ISDN, are technically possible with the HSCSD. However, circuit-switched services, such as the HSCSD, require two traffic channel (TCH) as a minimum per connection. Consequently, the HSCSD is advised as a good choice for applications with strict real-time constraint and bulk data transmission. A technological introduction in the GSM is the introduction of the GPRS. With the implementation of the GPRS, the GSM networks will be broadened for transmission as a packet and with direct interfunctioning Internet Protocol (IP) networks. The GPRS will support both data transmission types with the advantage of the last traffic channel network resources (frequencies) being able to be economically used. 


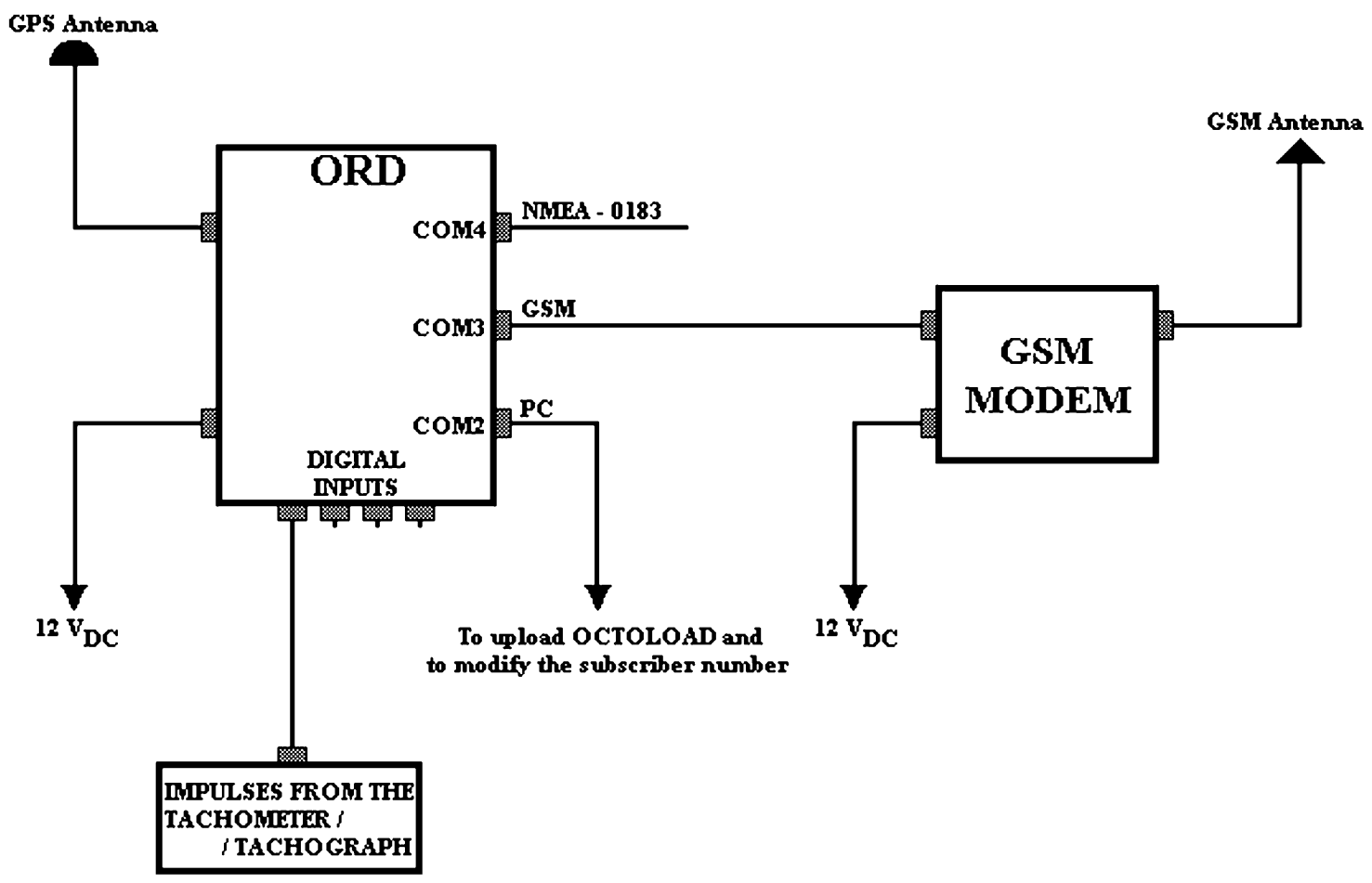

Fig. 21. Alternative connection diagram for RSC with GPS, in tunnels.

TABLE I

Status TABLE

\begin{tabular}{|c|c|c|c|}
\hline \multicolumn{2}{|c|}{ Inputs } & \multirow{2}{*}{$\begin{array}{c}\text { Output } \\
\text { (Through GSM) }\end{array}$} & \multirow[b]{2}{*}{ Comment } \\
\hline GPS Signal & $\begin{array}{c}\text { Impulses From the } \\
\text { Tachometer/Tachograph }\end{array}$ & & \\
\hline Exist & With impulses & $\begin{array}{l}\text { Send the GPS } \\
\text { coordinates }\end{array}$ & $\begin{array}{l}\text { Train moving in open } \\
\text { sky }\end{array}$ \\
\hline Exist & Without impulses & $\begin{array}{c}\text { Send the last } \\
\text { coordinate in memory }\end{array}$ & $\begin{array}{l}\text { Train stopped in open } \\
\text { sky }\end{array}$ \\
\hline Not exist & With impulses & $\begin{array}{l}\text { Send the coordinates } \\
\text { calculated with the help } \\
\text { of distance traveled }\end{array}$ & $\begin{array}{l}\text { Train moving in a } \\
\text { tunnel }\end{array}$ \\
\hline Not exist & Without impulses & $\begin{array}{c}\text { Send the last } \\
\text { coordinate in memory }\end{array}$ & $\begin{array}{l}\text { Train stopped in a } \\
\text { tunnel }\end{array}$ \\
\hline
\end{tabular}

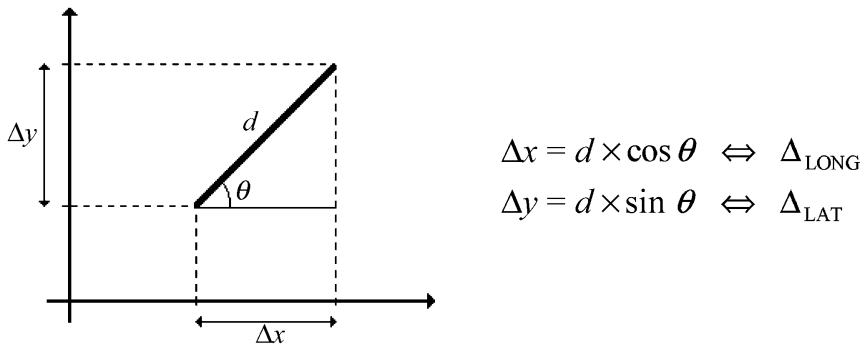

Fig. 22. Estimation of the variation of the distance ran.

Because the GPRS represents an opportunity to enhance the GSM-R, the greatest advantages and possible GPRS constraints in the railway environment are described below.

One of the main problems for the GSM is spectral limitation. The economic use of frequencies is of special importance to operator railway communications, since the International Union of Railways (UIC) frequency band is limited to $4 \mathrm{MHz}$ (20 digital network channels or, in circuit-commuting mode, 150 traffic channels). The GPRS, as a data packet service, restrains the use of the traffic channel at the time of the respective data packet transmission. Several users (up to 8) may simultaneously access a packet data traffic channel (PDTCH). This causes the GPRS to be exceptionally adequate to any application requiring burst-type data transmission, in a low data transmission rate, thus releasing the TCH for other applications. For bulk data transmission, the GPRS may offer a transmission speed up to $120 \mathrm{~kb} / \mathrm{s}$, using advanced reserves and channel codification systems with all eight available time slots for a transmission frequency. This transmission speed is consequently shared by all GRPS users present in the cell at that precise moment. Besides, this data transmission speed is affected by the cell/interference rate available in the radio call. Another GPRS advantage is the packet-commuting technology. The multisession capacity in a simple mobile avoids the need for several mobiles (one for each application). In a second phase, the GPRS will also provide the point-to-multipoint data transmission. In preview are the multicast, IP multicast transmission, and group data call. The only different type of data transmission with GSM broadcast capacities, the so-called SMS-cell broadcast service (SMS-CBS) does not provide 
TABLE II

ERROR CALCULATION

\begin{tabular}{c|c|c||}
\hline $\begin{array}{c}\text { Curvature } \\
\text { Radius } r \\
\text { (meter) }\end{array}$ & $\begin{array}{c}\text { Distance } \\
\text { Traveled in } \\
\text { Curve } d \\
\text { (meter) }\end{array}$ & $\begin{array}{c}\text { Error } \\
\text { (meter) }\end{array}$ \\
\hline \hline \multirow{2}{*}{350} & 100 & 15 \\
\hline \hline \multirow{2}{*}{1500} & 300 & 126 \\
\hline \hline \multirow{3}{*}{3000} & 100 & 4 \\
\cline { 2 - 4 } & 500 & 84 \\
\hline \hline \multirow{3}{*}{5000} & 100 & 2 \\
\cline { 2 - 4 } & 500 & 167 \\
\cline { 2 - 4 }
\end{tabular}

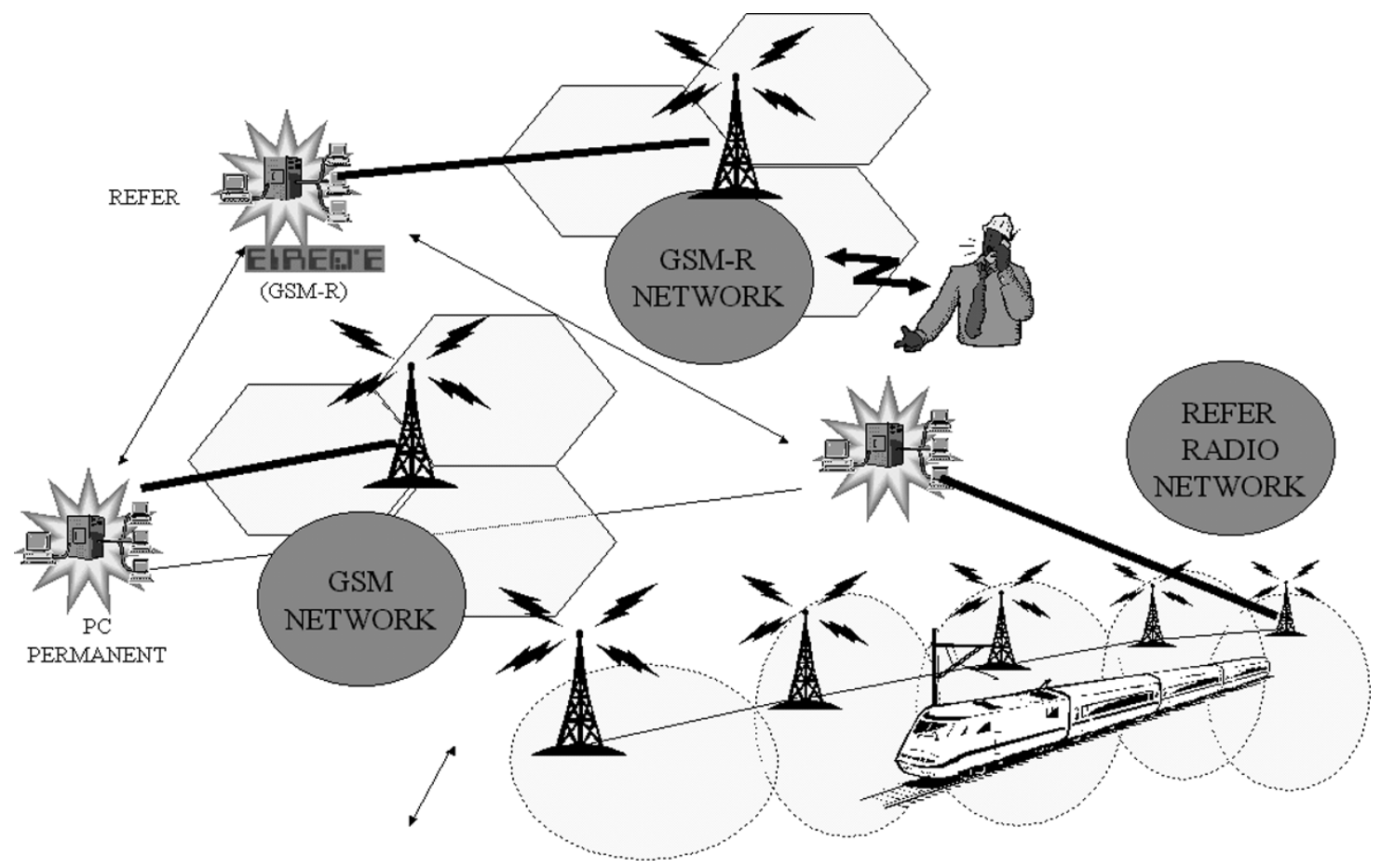

Fig. 23. Future of the railway communications in Portugal.

an equivalent data transmission speed and should not be used for applications of critical safety due to its nonreal-time behavior.

Apart from the critical real-time applications, all railway data transmission based applications can be supported by the GSM-R such as data transmission, e-mail services, mobile railway Intranet, mobile "office," broadcast information, vehicle and freight routing, and passenger services such as on-line ticket purchase/reservation.

As it has been said before, the future railway-signaling systems will be installed in all high-speed lines and in the main lines of each European country. Thus being, we have the medium/low-traffic regional lines where it is not viable to install these future systems. There, operators can improve the use of the GPS/GSM systems working together. The association of these two systems may become a very powerful tool, which will allow through the location of the train, via GPS, to activate voice and data transmission to specific track locations through the GSM system. Here are some examples.

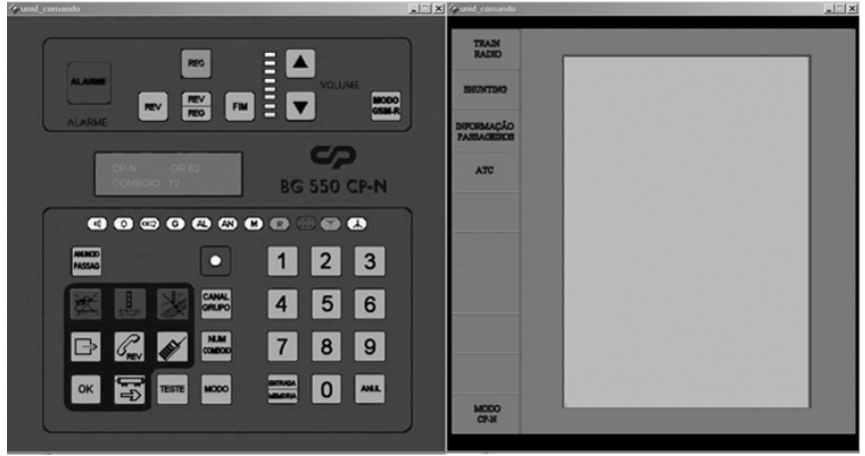

Fig. 24. BG550 CP-N and a possible desk GSM-R.

- The information on the closeness of a train, be it through sound or through data for the stops, stations, and level crossings in re- 


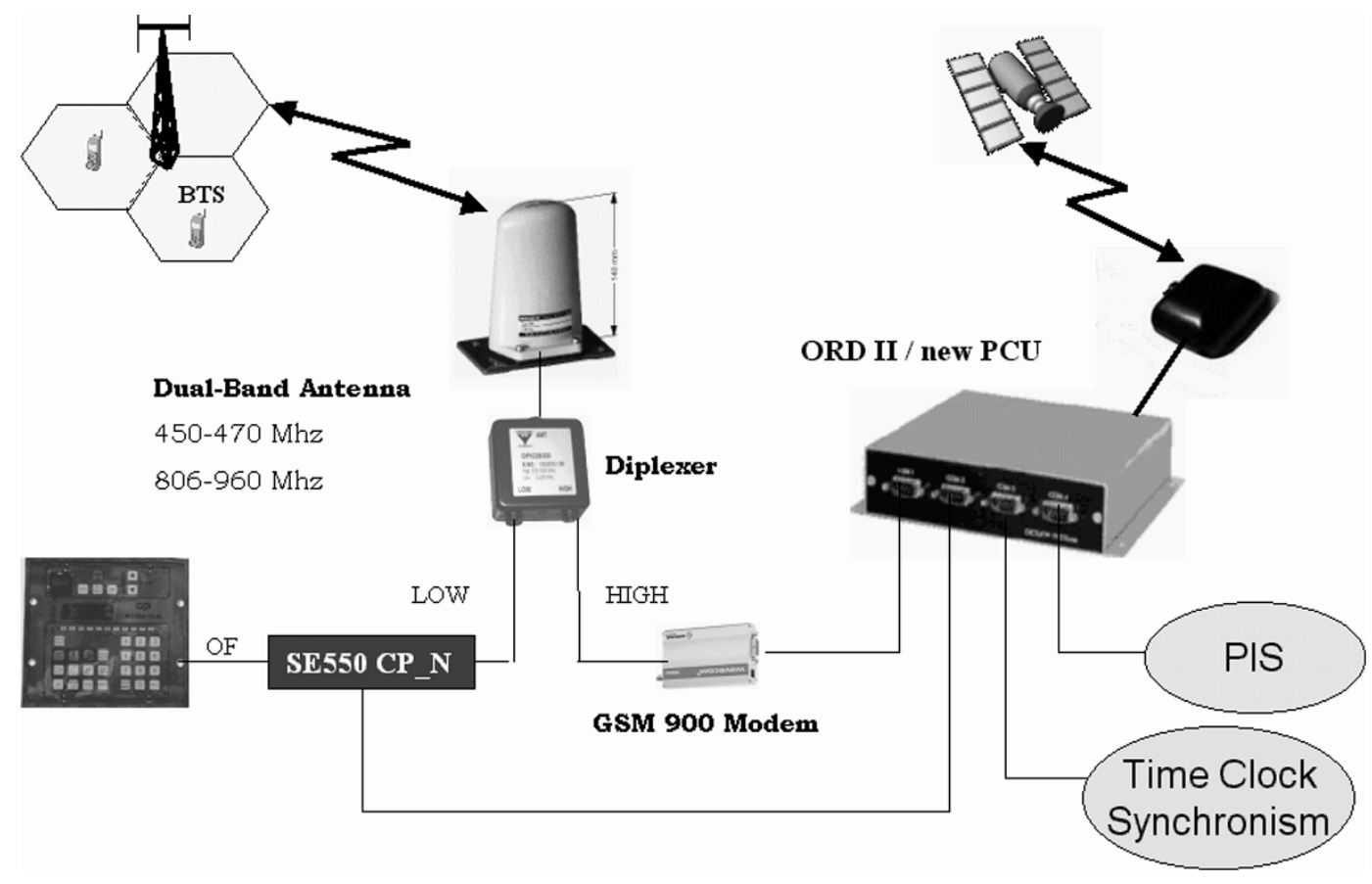

Fig. 25. Track-to-train communications near future.

gional lines. This sound information will also be useful for maintenance teams which are working along the track, namely, in places with difficult clearance for people and tools, such as tunnels and bridges.

- The automatic alteration of maximum speeds allowed, in variable information beacons, as are the cases of slackening caused by track maintenance works or by occasional alterations in track conditions.

- Possibility of monitoring and controlling train speed, through the speed provided by the GPS system, in tracks which are not equipped with the ATC system.

- Knowing the train location, we may set video cameras in front of the train to monitor the state of the track. This is particularly useful in mountains, where there is regular mass stone falling or avalanches and which may subsequently cause accidents involving some physical and material damage.

- Through the GSM system, we may transmit information data on a certain part of the track with running problems, for a control center, such as the weather information in steep slopes, where sometimes heavy freight trains are not able to climb or do it very slowly, causing great circulation delays. Also, the sending of surveillance information from driving cabs or train deposit parks may be very useful.

Currently, and relatively to track-to-train communications systems, it is worth guaranteeing compatibility with the GSM-R and there is still the possibility for a simultaneous operation of the future digital system and of the CP-N track-to-train communications system during the migration phase. That is, a system which is indifferent to the radio communication means used may operate supported by an analogical radio system (CP-N track-to-train communications, MPT 1327 trunking system, etc.) or a digital radio system (TETRA, GSM, GSM-R, or UMTS), only requiring for such to use the respective module and driver (Fig. 23). Consequently, an application for a demonstration has already been developed, for possible track-to-train communications + GSM + GSM-R clients, by way of demonstrating the possible potentialities of GSM integration and GSM-R migration. This application simulates telephony desks existing in trains, designated by BG550 CP-N, and a possible desk for the GSM-R (Fig. 24). This application is aimed at demonstrating the track-to-train communications desk and GSM-R desk functions in one desk only, which would start being displayed on a touch screen, and would have the chance to switch between desks. Therefore, we are currently working on future integrations in the track-to-train communications system (Fig. 25) such as GPRS, "point" and identification of the driving agents, time synchronism for ATC, power metering, new passenger information system (PIS), and telemetry and telesurveillance data transmission.

\section{CONCLUSION}

In this paper, a new system that is able not only to communicate with train but also to locate it with a great precision in real time, both inside and outside tunnels, has been described.

The solution presented here allows the immediate deployment of communication and tracking train systems with a minimum cost both in infrastructure and in maintenance. Thus is a viable solution to be used in secondary lines, where the available budget is limited.

That is why the contributions on this paper allows the deployment of an integrated system that simultaneously includes a radio communication system and a location solution in low-budget railways. The proposed network works as a redundant system when the traditional radio and tracking systems are available, but it will also work on secondary lines where no other system is available.

If we look at the fact that the overall system uses the train exclusively, then no infrastructure is really needed, cutting the deployment time and cost. Moreover, a low-budget system to track the trains inside tunnels was also presented again with a minimum infrastructure deployment.

\section{REFERENCES}

[1] Track to Train Communications System. Monte de Caparica, Portugal: NEC Portugal, 2000. 
[2] H. Hofestadt, "GSM-R: Global system for mobile radio communications for railways," in Int. Conf. Electric Railways in a United Europe, Amsterdam, The Netherlands, Mar. 1995, pp. 111-115.

[3] M. Leach, Ed., Train Control Systems. London, U.K.: Institute of Railway Signalling Engineers (IRSE), A\&C Black, 1993.

[4] M. Ikeda, "Characteristic of position detection and method of position correction by rotating axle," $Q$. Rep. RTRI Jpn., vol. 34, no. 4, pp. 270-276, 1993.

[5] G. A. Bates, "Use of GPS in a mobile data acquisition system," in IEEE Colloq. Dev. Use GPS Power Syst., London, U.K., Feb. 1994, pp. $2 / 1-2 / 3$.

[6] S. Fararooy, J. Allan, J. Jin, K. Maleki, and F. Pang, "Accurate train localisation in open space and tunnels," in Int. Conf. Public Transport Electronic Systems (Conf. Publ. No. 425), London, U.K., May 1996, pp. 25-29.
[7] “Full-scale Astree tests planned," Int. Railw. J., pp. 21-24, Nov. 1990.

[8] R. Mazl and L. Preucil, "Sensor data fusion for inertial navigation of trains in GPS-dark areas," in IEEE Intelligent Vehicles Symp. 2003 Proc., vol. 1, Piscataway, NJ, Jun. 2003, pp. 345-350.

[9] J. B.-Y. Tsui, Fundamentals of Global Positioning System Receivers: A Software Approach. New York: Wiley, 2000.

[10] E. D. Kaplan, Understanding GPS-Principles and Applications. Boston, MA: Artech House, 1996.

[11] C. Drane, Positioning Systems in Intelligent Transportation Systems. Boston, MA: Artech House, 1997.

[12] S. M. Redl, GSM and Personal Communications Handbook. Boston, MA: Artech House, 1998.

[13] J. Tisal, The GSM Network: GPRS Evolution: One Step Towards UMTS. Chichester, U.K.: Wiley, 2001. 